\title{
Successful early repair of a traumatic tracheoesophageal fistula after blunt chest trauma
}

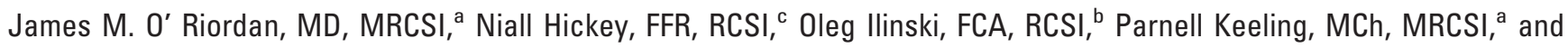
Thomas N. Walsh, MD, MRCSI, ${ }^{\text {a }}$ Dublin, Ireland

Supplemental material is available online.

$\mathrm{T}$

racheoesophageal fistula after blunt chest trauma is rare and usually presents after an asymptomatic interval. We report a case of acute traumatic tracheoesophageal fistula recognized immediately and treated successfully.

\section{Clinical Summary}

A 19-year-old man was the unrestrained driver of a car involved in a head-on collision with a wall. His car was not fitted with air bags, and he was found by the ambulance crew lying on the side of the road, having crawled out of the car. He arrived in the casualty department 30 minutes later. His pulse rate on arrival was 130 beats/min, blood pressure was $130 / 100 \mathrm{~mm} \mathrm{Hg}, \mathrm{PaO}_{2}$ was $8.0 \mathrm{kPa}$, oxygen saturation was $80 \%$ on $100 \% \mathrm{O}_{2}$, and Glasgow Coma Scale score was 11 of 15 . His main injuries on presentation were small bilateral pneumothorax, widespread subcutaneous emphysema, compound fractures of the right femur and right tibia, and closed fractures of the left femur and left fibula. Because of deteriorating oxygen saturation, he was intubated and ventilated. Bilateral chest drains were inserted, and his orthopedic injuries were stabilized with Thomas's splints.

A computed tomogram showed liver and splenic lacerations, blood in the peritoneal cavity, a pneumomediastinum, and massive subcutaneous emphysema (Figure 1). An esophageal or tracheal injury was suspected as a cause of the pneumomediastinum. An upper gastrointestinal endoscopy revealed a $5-\mathrm{cm}$ linear tear of the proximal esophagus between 20 and $25 \mathrm{~cm}$ extending into the trachea, with a tracheoesophageal fistula and a parallel tear extending into the mediastinum (Figure 2). Five hours after the accident, a right posterolateral thoracotomy was performed. The trachea was repaired with interrupted absorbable 4/0 Maxon sutures (United States Surgical, Tyco Healthcare). The 2 longitudinal tears in the esophagus $0.5 \mathrm{~cm}$ apart were converted to one by excluding the intervening mucosal strip at the expense of luminal narrowing. The esophageal repair was performed with inter-

From the Departments of Surgery, ${ }^{\mathrm{a}}$ Anaesthesia, ${ }^{\mathrm{b}}$ and Radiology, ${ }^{\mathrm{c}}$ Connolly Hospital, Blanchardstown, Dublin, Ireland.

Received for publication July 9, 2006; accepted for publication Aug 8, 2006.

Address for reprints: James O’Riordan, Connolly Hospital, Blanchardstown, Dublin 15, Ireland (E-mail: JamORiordan@rcsi.ie).

J Thorac Cardiovasc Surg 2006;132:1495-6

$0022-5223 / \$ 32.00$

Copyright $\odot 2006$ by The American Association for Thoracic Surgery doi:10.1016/j.jtcvs.2006.08.025

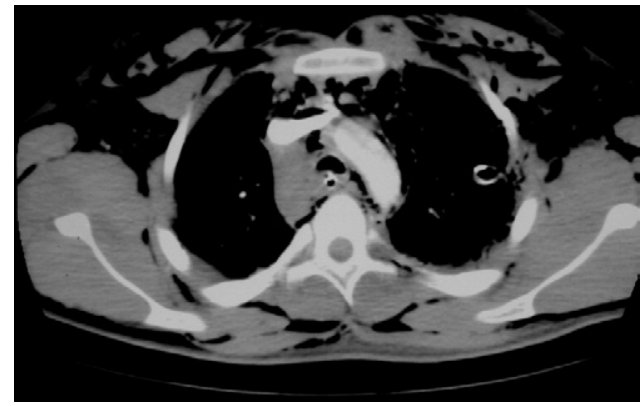

Figure 1. Computed tomogram of the thorax at the level of the aortic arch showing mediastinal hematoma, pneumomediastinum, and widespread subcutaneous emphysema.

rupted 4/0 Maxon sutures. This was reinforced by raising a large pleural patch and wrapping it around the entire repair. An interpositioned intercostal muscle flap was placed between the trachea and esophagus and secured to prevent recurrence of the fistula (Figure E1). Two pediatric endotracheal tubes were inserted into the right and left main bronchi to obviate the risk of disruption of the tracheal repair by the cuff of the endotracheal tube (Figure E2). Because these were too short to reach beyond the oral cavity, they were inserted through a formal tracheostomy.

Postoperatively, the patient was treated in the intensive care unit with nasoesophageal tube drainage, parenteral nutrition, and intravenous antibiotics, and his limb injuries were managed by the orthopedic team. He was maintained on bibronchial ventilation for 14 days, at which time repeat esophagoscopy and bronchoscopy showed that both repairs had healed. The pediatric endobronchial tubes were then changed to a single standard tracheostomy tube, which was removed on day 22. At this time, he was commenced on a light diet, which he tolerated without any problems. He required orthopedic, nutritional, and neurological rehabilitation and was discharged well to his home on day 65 .

\section{Discussion}

Tracheoesophageal fistula after blunt chest trauma is uncommon. Typically the patient is a young male subject with an elastic chest wall who is involved in a high-speed motor vehicle accident. ${ }^{1}$ Sudden deceleration causes anteroposterior compression to the anterior chest, with an explosive increase in esophageal and tracheal luminal pressure. In the majority of patients (60\%), symptoms develop 3 to 10 days after the initial blunt trauma. In this setting there is a posterior tracheal tear that initially seals, as well as an esophageal injury at the time of the initial trauma. The mucosal blood supply to the anterior portion of the esophagus is 


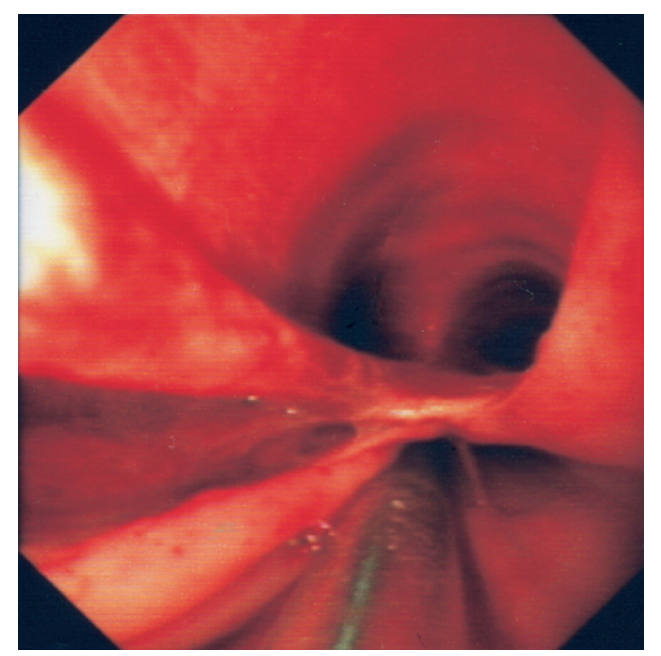

Figure 2. Upper gastrointestinal endoscopy showing the nasogastric tube in situ with two longitudinal tears in the esophagus and a clear view of the carina indicating a tracheoesophageal fistula.

impaired, resulting in delayed fistulization between the trachea and the esophagus. ${ }^{2}$

An acute communication between the trachea and esophagus, as in this case, is extremely uncommon after blunt chest trauma, and we could only find 7 such cases reported in the literature..$^{3,4}$ This is probably because of the high incidence of associated mediastinal injuries that prove fatal. By definition, a full-thickness injury of the trachea and esophagus occurs at the time of impact.
Clinical signs include the "breathing-bag sign," subcutaneous emphysema, pneumothorax, or pneumomediastinum. ${ }^{5}$ Computed tomography, upper gastrointestinal endoscopy, esophagography, and bronchoscopy are useful adjuncts to assist in the diagnosis.

A right thoracotomy is the preferred approach to fistula repair by using interrupted absorbable sutures to repair both the trachea and esophagus. It is essential to use either pleura or intercostal muscle (we used both) interposed between the suture lines to prevent recurrence. We would also suggest ventilating below the tracheal suture line. We used 2 cuffed pediatric size 5 endotracheal tubes inserted into each bronchus through a tracheostomy to compensate for the reduced length.

In summary, an acute tracheoesophageal fistula after blunt chest trauma is an extremely uncommon injury. A high index of suspicion is required because early diagnosis and repair are essential for a successful outcome.

\section{References}

1. Karunaratne BL, Gooneratne PA, Wijesekara S, et al. Acquired tracheoesophageal fistula following blunt trauma to the chest. Asian Cardiovasc Thorac Ann. 2002;10:349-50.

2. Weber SM, Schurr MJ, Pellet JR. Delayed presentation of a tracheoesophageal fistula after blunt chest trauma. Ann Thorac Surg. 1996;62: $1850-2$.

3. Fitzpatrick BT, O'Grady JF, Sayed K, et al. Acute tracheoesophageal communication: a diagnostic sign for an unusual injury. Ir Med J. 1983;76:421-2.

4. Tsai FC, Lin PJ, Wu YV, et al. Traumatic aortic arch transection with supracarinal tracheoesophageal fistula: case report. J Trauma. 1999;46: 951-3.

5. Rampaul RS, Naraynsingh V, Dean VS. Tracheoesophageal fistula following blunt chest trauma: diagnosis in the ICU-the "breathing bag sign." Chest. 1999;116:267. 


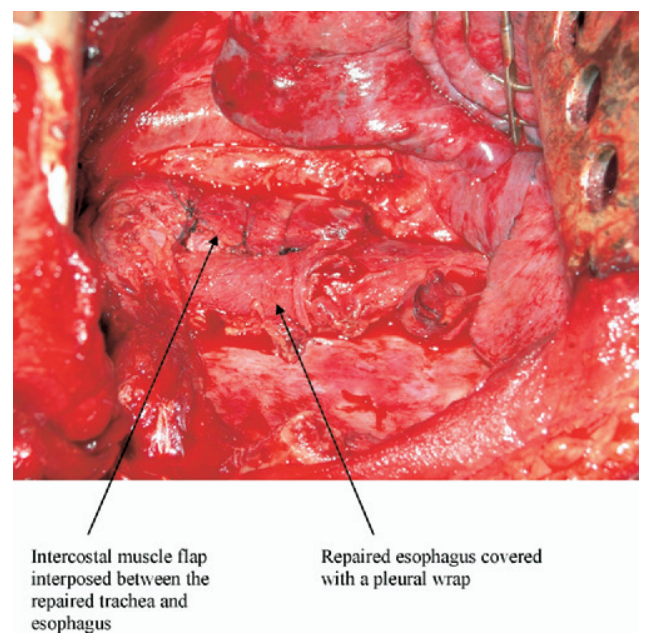

Figure E1. Intraoperative photograph taken through the right chest showing the trachea and esophagus having been repaired. The esophagus is reinforced with a pleural patch, and an intercostal muscle flap is placed between it and the trachea.

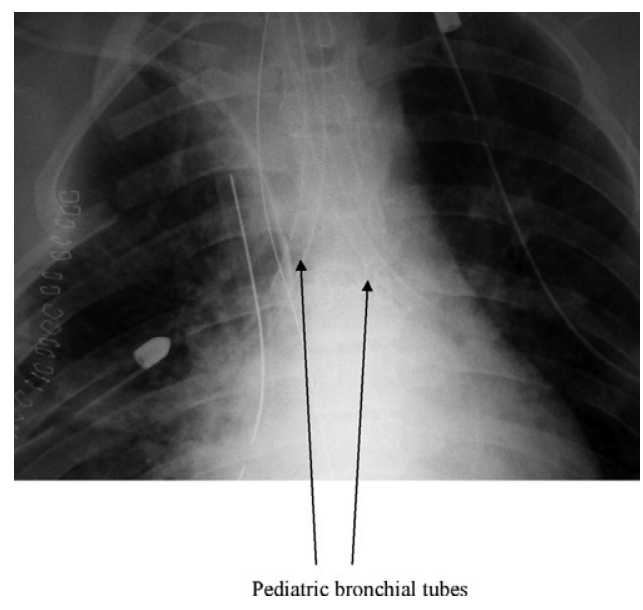

Figure E2. Portable chest radiograph taken on the first postoperative day showing the 2 pediatric bronchial tubes in situ in each main bronchus below the tracheal suture line. 